\title{
A search for dark matter in Triangulum II with the MAGIC telescopes
}

MAGIC Collaboration: V. A. Acciari ${ }^{\mathrm{a}}$, S. Ansoldi ${ }^{\mathrm{b}, \mathrm{w}}$, L. A. Antonelli ${ }^{\mathrm{c}}$, A. Arbet Engels ${ }^{\mathrm{d}}$, D. Baack $^{\mathrm{e}}$, A. Babić ${ }^{f}$, B. Banerjee ${ }^{g}$, U. Barres de Almeida ${ }^{\mathrm{h}}$, J. A. Barrio ${ }^{\mathrm{i}}, \mathrm{J}_{\text {. Becerra González }}^{\mathrm{a}}$, W. Bednarek ${ }^{\mathrm{j}}$, L. Bellizzi $^{\mathrm{k}}$, E. Bernardini ${ }^{1, p}$, A. Berti ${ }^{\mathrm{m}}$, J. Besenrieder ${ }^{\mathrm{n}}$, W. Bhattacharyya ${ }^{\mathrm{l}}$, C. Bigongiari ${ }^{\mathrm{c}}$, A. Biland ${ }^{\mathrm{d}}$,

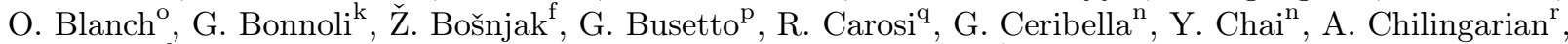

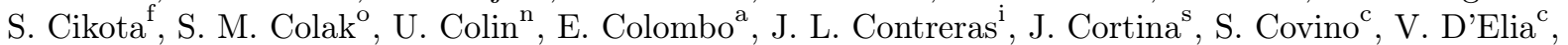
P. Da Vela ${ }^{\mathrm{q}}$, F. Dazzi ${ }^{\mathrm{c}}$, A. De Angelis ${ }^{\mathrm{p}}$, B. De Lotto ${ }^{\mathrm{b}}$, M. Delfino ${ }^{\mathrm{o}, \mathrm{z}}$, J. Delgado ${ }^{\mathrm{o}, \mathrm{z}}$, D. Depaoli $^{\mathrm{m}}$, F. Di Pierro $^{\mathrm{m}}$, L. Di Venere ${ }^{\mathrm{m}}$, E. Do Souto Espiñeira ${ }^{\mathrm{o}}$, D. Dominis Prester ${ }^{\mathrm{f}}$, A. Donini ${ }^{\mathrm{b}}$, D. Dorner ${ }^{\mathrm{t}}$, M. Doro ${ }^{\mathrm{p}, *}$, D. Elsaesser ${ }^{\mathrm{e}}$, V. Fallah Ramazani ${ }^{\mathrm{u}}$, A. Fattorini ${ }^{\mathrm{e}}$, G. Ferrara ${ }^{\mathrm{c}}$, D. Fidalgo ${ }^{\mathrm{i}}$, L. Foffano ${ }^{\mathrm{p}}$, M. V. Fonseca ${ }^{\mathrm{i}}$,

L. Font ${ }^{\mathrm{v}}$, C. Fruck ${ }^{\mathrm{n}}$, S. Fukami ${ }^{\mathrm{w}}$, R. J. García López ${ }^{\mathrm{a}}$, M. Garczarczyk ${ }^{1}$, S. Gasparyan ${ }^{\mathrm{r}}$, M. Gaug $^{\mathrm{v}}$, N. Giglietto ${ }^{\mathrm{m}}$, F. Giordano ${ }^{\mathrm{m}}$, P. Gliwny ${ }^{\mathrm{j}}$, N. Godinović ${ }^{\mathrm{f}}$, D. Green ${ }^{\mathrm{n}}$, J. G. Green ${ }^{\mathrm{c}, *}$, D. Guberman ${ }^{\mathrm{o}}$,

D. Hadasch ${ }^{\mathrm{w}}$, A. Hahn ${ }^{\mathrm{n}}$, J. Herrera ${ }^{\mathrm{a}}$, J. Hoang ${ }^{\mathrm{i}}$, D. Hrupec ${ }^{\mathrm{f}}$, M. Hütten ${ }^{\mathrm{n}}$, T. Inada ${ }^{\mathrm{w}}$, S. Inoue $^{\mathrm{w}}$, K. Ishio ${ }^{\mathrm{n}}$, Y. Iwamura ${ }^{w}$, L. Jouvin ${ }^{\circ}$, D. Kerszberg ${ }^{\mathrm{o}}$, H. Kubow ${ }^{\mathrm{w}}$, J. Kushida ${ }^{\mathrm{w}}$, A. Lamastra ${ }^{\mathrm{c}}$, D. Lelas ${ }^{\mathrm{f}}$, F. Leone ${ }^{\mathrm{c}}$,

E. Lindfors ${ }^{\mathrm{u}}$, S. Lombardi ${ }^{\mathrm{c}}$, F. Longo ${ }^{\mathrm{b}, \mathrm{aa}}$, M. López ${ }^{\mathrm{i}}$, R. López-Coto ${ }^{\mathrm{p}}$, A. López-Oramas ${ }^{\mathrm{a}}$, S. Loporchio ${ }^{\mathrm{m}}$, B. Machado de Oliveira Fraga ${ }^{\mathrm{h}}$, C. Maggiov , P. Majumdar ${ }^{\mathrm{g}}$, M. Makariev $^{\mathrm{x}}$, M. Mallamaci ${ }^{\mathrm{p}}$, G. Maneva ${ }^{\mathrm{x}}$, M. Manganaro ${ }^{f}$, K. Mannheim ${ }^{\mathrm{t}}$, L. Maraschi ${ }^{\mathrm{c}}$, M. Mariotti $^{\mathrm{p}}$, M. Martínez $^{\mathrm{o}}$, D. Mazin ${ }^{\mathrm{n}, \mathrm{w}}$, S. Mićanović ${ }^{\mathrm{f}}$, D. Miceli ${ }^{\mathrm{b}}$, T. Miener ${ }^{\mathrm{i}}$, M. Minev ${ }^{\mathrm{x}}$, J. M. Miranda ${ }^{\mathrm{k}}$, R. Mirzoyan ${ }^{\mathrm{n}}, \mathrm{E}_{\text {E. Molina }}^{\mathrm{y}}$, A. Moralejo ${ }^{\mathrm{o}}$,

D. Morcuende ${ }^{\mathrm{i}}$, V. Moreno ${ }^{\mathrm{v}}$, E. Moretti ${ }^{\mathrm{o}}$, P. Munar-Adrover ${ }^{\mathrm{v}}$, V. Neustroev ${ }^{\mathrm{u}}$, C. Nigro ${ }^{\mathrm{l}}$, K. Nilsson ${ }^{\mathrm{u}}$,

D. Ninci ${ }^{\mathrm{o}}$, K. Nishijima ${ }^{\mathrm{w}}$, K. Noda ${ }^{\mathrm{w}}$, L. Nogués ${ }^{\mathrm{o}}$, S. Nozaki ${ }^{\mathrm{w}}$, S. Paiano ${ }^{\mathrm{p}}$, J. Palacio ${ }^{\mathrm{o}, *}$, M. Palatiello ${ }^{\mathrm{b}}$,

D. Paneque ${ }^{n}$, R. Paoletti ${ }^{\mathrm{k}}$, J. M. Paredes ${ }^{\mathrm{y}}$, P. Peñil ${ }^{\mathrm{i}}$, M. Peresano ${ }^{\mathrm{b}}$, M. Persic $^{\mathrm{b}, a b}$, P. G. Prada Moroni ${ }^{\mathrm{q}}$, E. Prandini ${ }^{\mathrm{p}}$, I. Puljak ${ }^{\mathrm{f}}$, W. Rhode ${ }^{\mathrm{e}}$, M. Ribó ${ }^{\mathrm{y}}$, J. Rico ${ }^{\circ}$, C. Righi ${ }^{\mathrm{c}}$, A. Rugliancich ${ }^{\mathrm{q}}$, L. Saha $^{\mathrm{i}}$, N. Sahakyan ${ }^{r}$, T. Saitow ${ }^{w}$, S. Sakurai ${ }^{w}$, K. Sataleckal, F. G. Saturni ${ }^{\text {c,* }}$, K. Schmidt ${ }^{\mathrm{e}}$, T. Schweizer ${ }^{\mathrm{n}}$, J. Sitarek ${ }^{j}$, I. Śnidarić ${ }^{\mathrm{f}}$, D. Sobczynska ${ }^{\mathrm{j}}$, A. Somero ${ }^{\mathrm{a}}$, A. Stamerra ${ }^{c}$, D. Strom ${ }^{\mathrm{n}}$, Y. Suda ${ }^{\mathrm{n}}$, T. Surić ${ }^{\mathrm{f}}$, M. Takahashi ${ }^{\mathrm{w}}$, F. Tavecchio ${ }^{\mathrm{c}}$, P. Temnikov ${ }^{\mathrm{x}}$, T. Terzić ${ }^{\mathrm{f}}$, M. Teshima ${ }^{\mathrm{n}, \mathrm{w}}$, N. Torres-Albà ${ }^{\mathrm{y}}$, L. Tosti $^{\mathrm{m}}$,

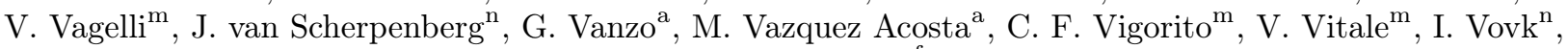
M. Will ${ }^{\mathrm{n}}$, D. Zarić ${ }^{\mathrm{f}}$

\footnotetext{
${ }^{a}$ Inst. de Astrofísica de Canarias, E-38200 La Laguna, and Universidad de La Laguna, Dpto. Astrofísica, E-38206 La Laguna, Tenerife, Spain

${ }^{b}$ Università di Udine, and INFN Trieste, I-33100 Udine, Italy

${ }^{c}$ National Institute for Astrophysics (INAF), I-00136 Rome, Italy ${ }^{d}$ ETH Zurich, CH-8093 Zurich, Switzerland

${ }^{e}$ Technische Universität Dortmund, D-44221 Dortmund, Germany

${ }^{f}$ Croatian Consortium: University of Rijeka, Department of Physics, 51000 Rijeka; University of Split - FESB, 21000 Split; University of Zagreb - FER, 10000 Zagreb; University of Osijek, 31000 Osijek; Rudjer Boskovic Institute, 10000 Zagreb, Croatia

${ }^{g}$ Saha Institute of Nuclear Physics, HBNI, 1/AF Bidhannagar, Salt Lake, Sector-1, Kolkata 700064, India

${ }^{h}$ Centro Brasileiro de Pesquisas Físicas (CBPF), 22290-180 URCA, Rio de Janeiro (RJ), Brasil

${ }^{i} I P A R C O S$ Institute and EMFTEL Department, Universidad Complutense de Madrid, E-28040 Madrid, Spain

${ }^{j}$ University of Łódż, Department of Astrophysics, PL-90236 Łódż, Poland

${ }^{k}$ Università di Siena and INFN Pisa, I-53100 Siena, Italy

${ }^{l}$ Deutsches Elektronen-Synchrotron (DESY), D-15738 Zeuthen, Germany

${ }^{m}$ Istituto Nazionale Fisica Nucleare (INFN), 00044 Frascati (Roma) Italy

${ }^{n}$ Max-Planck-Institut für Physik, D-80805 München, Germany

${ }^{\circ}$ Institut de Física d'Altes Energies (IFAE), The Barcelona Institute of Science and Technology (BIST), E-08193 Bellaterra (Barcelona), Spain

${ }^{p}$ Università di Padova and INFN, I-35131 Padova, Italy

${ }^{q}$ Università di Pisa, and INFN Pisa, I-56126 Pisa, Italy

${ }^{r}$ The Armenian Consortium: ICRANet-Armenia at NAS RA, A. Alikhanyan National Laboratory

${ }^{s}$ Centro de Investigaciones Energéticas, Medioambientales y Tecnológicas, E-28040 Madrid, Spain

${ }^{t}$ Universität Würzburg, D-97074 Würzburg, Germany

${ }^{u}$ Finnish MAGIC Consortium: Finnish Centre of Astronomy with ESO (FINCA), University of Turku, FI-20014 Turku, Finland; Astronomy Research Unit, University of Oulu, FI-90014 Oulu, Finland

${ }^{v}$ Departament de Física, and CERES-IEEC, Universitat Autònoma de Barcelona, E-08193 Bellaterra, Spain
} 


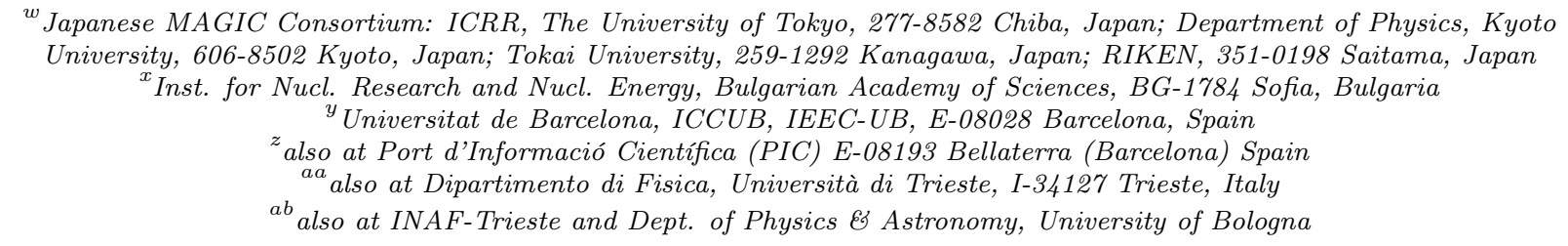

\begin{abstract}
We present the first results from very-high-energy observations of the dwarf spheroidal satellite candidate Triangulum II with the MAGIC telescopes from 62.4 hours of good-quality data taken between August 2016 and August 2017. We find no gamma-ray excess in the direction of Triangulum II, and upper limits on both the differential and integral gamma-ray flux are presented. Currently, the kinematics of Triangulum II are affected by large uncertainties leading to a bias in the determination of the properties of its dark matter halo. Using a scaling relation between the annihilation $J$-factor and heliocentric distance of well-known dwarf spheroidal galaxies, we estimate an annihilation $J$-factor for Triangulum II for WIMP dark matter of $\log \left[J_{\text {ann }}\left(0.5^{\circ}\right) / \mathrm{GeV}^{2} \mathrm{~cm}^{-5}\right]=19.35 \pm 0.37$. We also derive a dark matter density profile for the object relying on results from resolved simulations of Milky Way sized dark matter halos. We obtain 95\% confidence-level limits on the thermally averaged annihilation cross section for WIMP annihilation into various Standard Model channels. The most stringent limits are obtained in the $\tau^{-} \tau^{+}$final state, where a cross section for annihilation down to $\left\langle\sigma_{\text {ann }} v\right\rangle=3.05 \times 10^{-24} \mathrm{~cm}^{3} \mathrm{~s}^{-1}$ is excluded.
\end{abstract}

Keywords: dark matter, indirect searches, dwarf spheroidal satellite galaxies, Imaging Air Cherenkov Telescopes, Triangulum II

\title{
1. Introduction
}

The dark matter (DM) paradigm arises from observational evidence which shows the Standard Model (SM) of particle physics cannot entirely explain the gravitational effects on astrophysical systems observed at all cosmological scales, from Milky Way (MW) satellite dwarf spheroidal galaxies (dSphs) to clusters of galaxies [1, 2]. DM is expected to represent nearly $85 \%$ of the total matter content of our universe, however its true nature remains elusive. The existence of one or more new massive particles not belonging to the SM is a well-favored explanation. Experimental evidence constrains the DM particle to be stable on cosmological timescales, electrically neutral, and non-baryonic. Weakly interacting massive particles (WIMPs) fulfill all of these requirements and are among the best-motivated DM candidates [3, 4. Expected to have masses in the range of a few $\mathrm{GeV}$ to a few $\mathrm{TeV}$, WIMPs interact with SM particles at most on the weak scale, and could explain the observed relic density of $\Omega_{D M}=0.259 \pm 0.006$ [5]. WIMP particles can interact and produce various SM particles, possibly including gamma rays that could be detected by ground- and space-based observatories. In spite of the various efforts that have been performed, no evidence for the existence of DM particles has been found.

The Florian Goebel Major Atmospheric Gamma-ray Imaging Cherenkov (MAGIC) telescope is a pair of Imaging Atmospheric Cherenkov Telescopes (IACTs), each $17 \mathrm{~m}$ in diameter, located at the Roque de los Muchachos Observatory (Spain). The system is sensitive in the very-high-energy (VHE) gamma-ray regime. MAGIC is able to detect gamma rays via the Cherenkov light produced during atmospheric showers initiated when VHE photons interact with the Earth's atmosphere. For low zenith angle observations, MAGIC has standard trigger threshold of $\sim 50 \mathrm{GeV}$, a $68 \%$ containment angular point spread function $(\mathrm{PSF})$ of $\sim 0.1$

\footnotetext{
*Corresponding authors: jarred.green@inaf.it, francesco.saturni@inaf.it, jpalacio@ifae.es, michele.doro@unipd.it
} 
deg for gamma rays of $\sim 200 \mathrm{GeV}$, and an energy resolution of $16 \%$ [ 6], rendering the instrument particularly well-suited to perform indirect DM searches. Among the most promising astrophysical objects for indirect DM searches with IACTs are dSphs [7, 8]. These compact galaxies are gravitationally bound to the MW and located in its galactic halo. They have very low luminosities, few member stars, no gas or dust, and tend to be approximately spheroidal in shape. In addition, a low astrophysical background makes them optimal targets for searches in the gamma-ray regime [9]. They have among the highest mass-to-light ratios (M/L) of any known astrophysical object, and many are found relatively nearby (within $250 \mathrm{kpc}$ of the center of the MW).

The first VHE indirect DM search based on the observation campaign of Triangulum II (Tri II), a relatively nearby and a potential DM-dense dSph [10, 11, was carried out with the MAGIC Telescopes and is reported in this paper. These observations are part of a multi-year MAGIC campaign of dSphs [12, 13, which follows a common analysis likelihood framework [14. The main difference with respect to previous MAGIC dSph studies lies in the determination of the $J$-factor and emission morphology of Tri II, due to an absence of sufficient stellar kinematic data.

\section{Standard gamma-ray analysis of Triangulum II}

Triangulum II is an ultra-faint MW satellite discovered in 2015 as part of the Pan-STARRS survey 15. It is located at RA(J2000) $02 \mathrm{~h} 13 \mathrm{~m} 17.4 \mathrm{~s}$, Dec(J2000) $+36^{\circ} 10^{\prime} 42.4^{\prime \prime}$, with an absolute magnitude of $M_{v}=-1.8 \pm 0.5$ at a heliocentric distance of $d=30 \pm 2 \mathrm{kpc}$ [16]. A first spectroscopic study, based on six member stars, predicted Tri II to have a M/L of $3600_{-2100}^{+3500} M_{\odot} / L_{\odot}$ and one of the highest DM concentrations of any galaxy ever found [10].

An observation campaign of Tri II was subsequently carried out with the MAGIC telescope. MAGIC collected 62.4 h of good-quality data between August 2016 and August 2017 as part of a multi-year campaign to study different dSphs [12, 13. The Tri II data were collected at low zenith angles, between $\sim 5^{\circ}$ and $\sim 35^{\circ}$. The observations were carried out in 'wobble' mode, where the target was tracked with a $0.4^{\circ}$ offset from the center of the camera [17, allowing for the simultaneous measurement of both the source ('ON') and the background control ('OFF') regions. Two telescope pointings were used, lying on an axis inclined $148^{\circ}$ with respect to the line of constant declination at the Tri II position (which prevented the relatively bright nearby star $\beta$ Trianguli, $m=3.02$, from illuminating the trigger area of the MAGIC cameras [6]).

More recent kinematic studies of Tri II, carried out after the MAGIC observation campaign, indicate that Tri II might not be in dynamical equilibrium [11. Further spectral measurements with Keck/DEIMOS of 13 member stars hint at the fact that the object is currently in the process of disruption and is not virialized as a result, or possibly embedded in a stellar stream [18, 19]. Further studies suggest that one of the original stars used in kinematic calculations was actually a binary system, which artificially increased the total velocity dispersion thus wrongfully boosting the estimated DM density in [11.

The data calibration and analysis was carried out using the standard MAGIC Analysis and Reconstruction Software (MARS) [20]. The total sample of data underwent a selection process for excellent atmospheric conditions, quantified by a proprietary LIDAR sensing instrument [21]. Events surviving the aforementioned data selection criteria are assigned an estimated energy and direction, as well as a gamma/hadron discriminator called "hadronness" or $h . h$ is a computed by the comparison of real data with dedicated Monte Carlo (MC) gamma-ray simulations using the Random Forest method [22].

In Figure 1, following the standard low-energy analysis of MAGIC, we show the distribution of the number of gamma-like events as a function of $\theta^{2}$, the squared angular distance between the reconstructed event direction and the nominal position of the target, around the ON and OFF regions No excess gamma-ray signal is detected over the background in the direction of Tri II.

A significance map centered in the target sky position of Tri II is shown in Figure 2. This sky-map is generated using a test statistic according to [23] (Eq. 17) and is applied on a smoothed, modeled background estimation [24]. Again, no hint of emission is seen from the region of Tri II.

\footnotetext{
${ }^{1}$ Distribution of events in the OFF region has been scaled to match the one from the ON region at distances much larger than our fiducial signal region in $\theta^{2}$.
} 


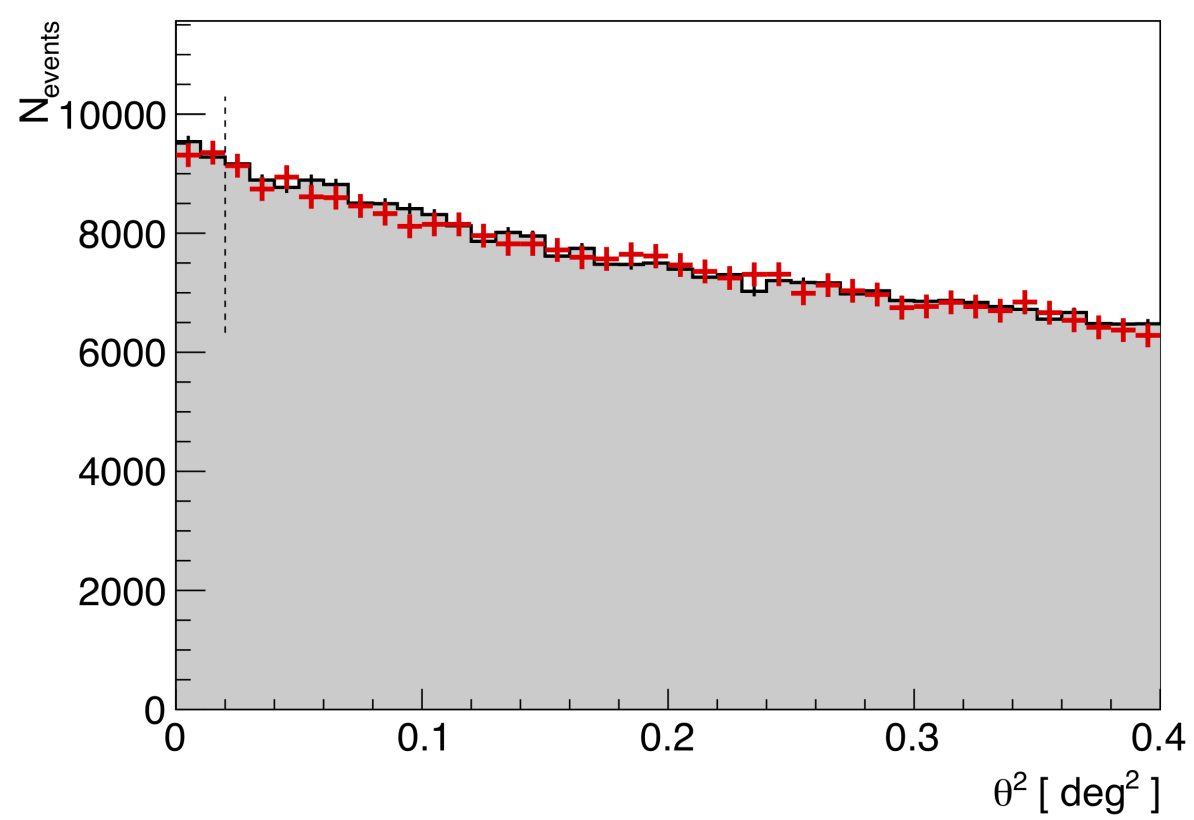

Figure 1: The $\theta^{2}$ distribution of gamma-like events in the ON (red crosses) and OFF (shaded) regions for Tri II. The vertical dashed line at $\theta^{2}=0.02 \mathrm{deg}^{2}$ shows the border of the fiducial signal region defined for a point-like source. The distribution of events in the OFF region has been scaled to match that of the ON region at distances far outside this fiducial signal region. There is no significant detection of gamma-ray signal over the background.

\subsection{Gamma-ray flux limits}

We derive $95 \%$ confidence level (CL) upper limits (UL) on the gamma-ray flux from the region of Tri II. We follow the same framework as adopted from the MAGIC Segue I dSph analysis [12, that made use of power law spectra with 7 different values of the spectral index $\Gamma$ ranging between -1 and -2.4 . We select events based on $\mathrm{h}$ retaining $80 \% \mathrm{MC}$ gamma rays in each energy bin and define a fiducial signal region until $\theta^{2}=0.02 \mathrm{deg}^{2}$ (these cuts are used through the rest of this paper unless otherwise specified). The differential flux ULs in the energy range $100 \mathrm{GeV}$ to $10 \mathrm{TeV}$ are shown for each assumed spectral index in Figure 3, and the obtained ULs are compared, for reference, to the Crab Nebula flux as measured by MAGIC [25]. The $\mathrm{x}$-axis is defined in terms of the pivot energy $E^{*}$, which is calculated for each energy bin (see Equation A.2 of [12]).

The integral flux ULs for gamma rays with $E>E_{\mathrm{th}}$ are shown in Figure 4 , for various assumed energy thresholds $E_{\mathrm{th}}$. We note that, as expected, the integral ULs are more dependent on the assumed spectral index and are more sensitive to statistical fluctuations than the differential limits. To quantify this effect, we also calculate the expected ULs under a 'null-hypothesis' $N_{\mathrm{ex}}^{\mathrm{UL}}\left(E>E_{\mathrm{th}}\right)=0$, shown as dashed lines in Figure 4. In this case, a significance of zero (i.e. when the signal region of the $\theta^{2}$ plot contains a number of events in the ON region equal to the scaled number of events in the OFF region) is assumed for each value of $\Gamma$ and $E_{\mathrm{th}}$. The numerical values of the obtained flux ULs of Figure 3 and Figure 4 are shown in Appendix A.

\section{Dark matter searches in Triangulum II}

We perform a dedicated analysis of gamma-ray signatures of DM particles annihilating into SM pairs, taking advantage of the MAGIC observations of Tri II presented in Section 2. The detection of a potential signal in the gamma-ray energy window $100 \mathrm{GeV}-100 \mathrm{TeV}$ would provide information both on the nature of the DM progenitor particles and on the DM distribution in the direction of the source. In the case that 


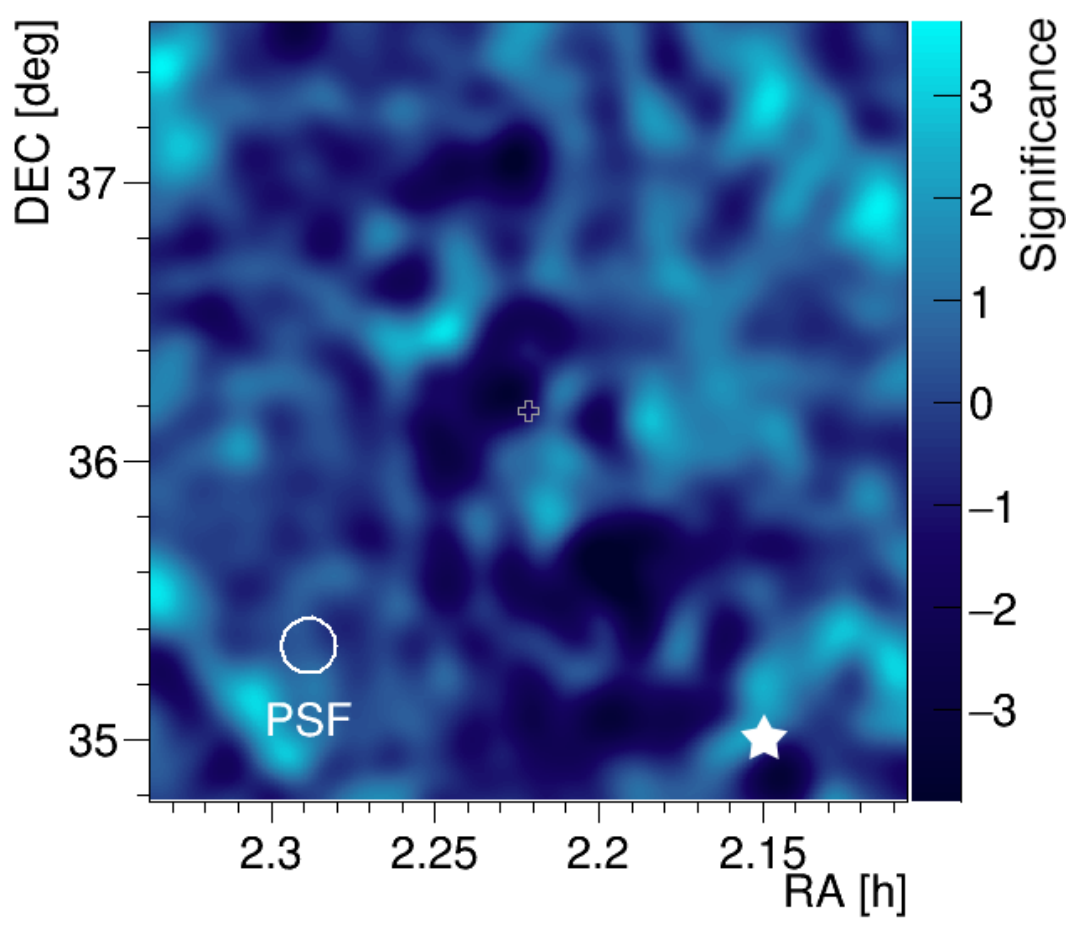

Figure 2: Significance sky-map centered at the Tri II sky position (white cross). No significant gamma-ray signal is detected over the background. The color scale represents the test significance. The white circle shows the average MAGIC PSF $(68 \%$ containment) for the event selection used in the analysis (see Section 2.1). The location of the star $\beta$ Trianguli is shown as a white star.

no signal is detected, constraints on the annihilation cross section of DM can be obtained, allowing for an exploration of the parameter space of potential candidate particles.

The expected gamma-ray flux from DM annihilation in nearby sources can be expressed as the product of two terms:

$$
\frac{d \Phi_{\gamma}}{d E}=\frac{d \Phi_{\gamma}^{P P}}{d E} J_{\mathrm{ann}}(\Omega)
$$

where $d \Phi_{\gamma}^{P P} / d E$ and $J_{\text {ann }}(\Omega)$ are usually referred to as the particle physics factor and the astrophysical factor, respectively. The factor $d \Phi_{\gamma}^{P P} / d E$ contains all information related to the particle behavior:

$$
\frac{d \Phi_{\gamma}^{P P}}{d E}=\frac{\left\langle\sigma_{\mathrm{ann}} v\right\rangle}{8 \pi m_{D M}^{2}} \frac{d N_{\gamma}}{d E},
$$

where $\left\langle\sigma_{\text {ann }} v\right\rangle$ is the thermally averaged DM annihilation cross section, $m_{D M}$ is the DM particle mass, and $d N_{\gamma} / d E$ provides the average spectrum of photons expected per each annihilation interaction. The astrophysical factor $J_{\text {ann }}(\Omega)$, also known as the $J$-factor, contains information on how DM is distributed in the source with respect to earth [26], and is calculated via the line-of-sight (l.o.s.) integral of the square of the DM density distribution:

$$
J_{\text {ann }}(\Delta \Omega)=\int_{\Delta \Omega} \int_{l o s} \rho^{2}(r(l)) d l d \Omega,
$$

where $l, \rho(r(l))$ and $\Delta \Omega$ are the l.o.s. element, density profile of DM within the source, and the solid angle of the integrated region, respectively. 


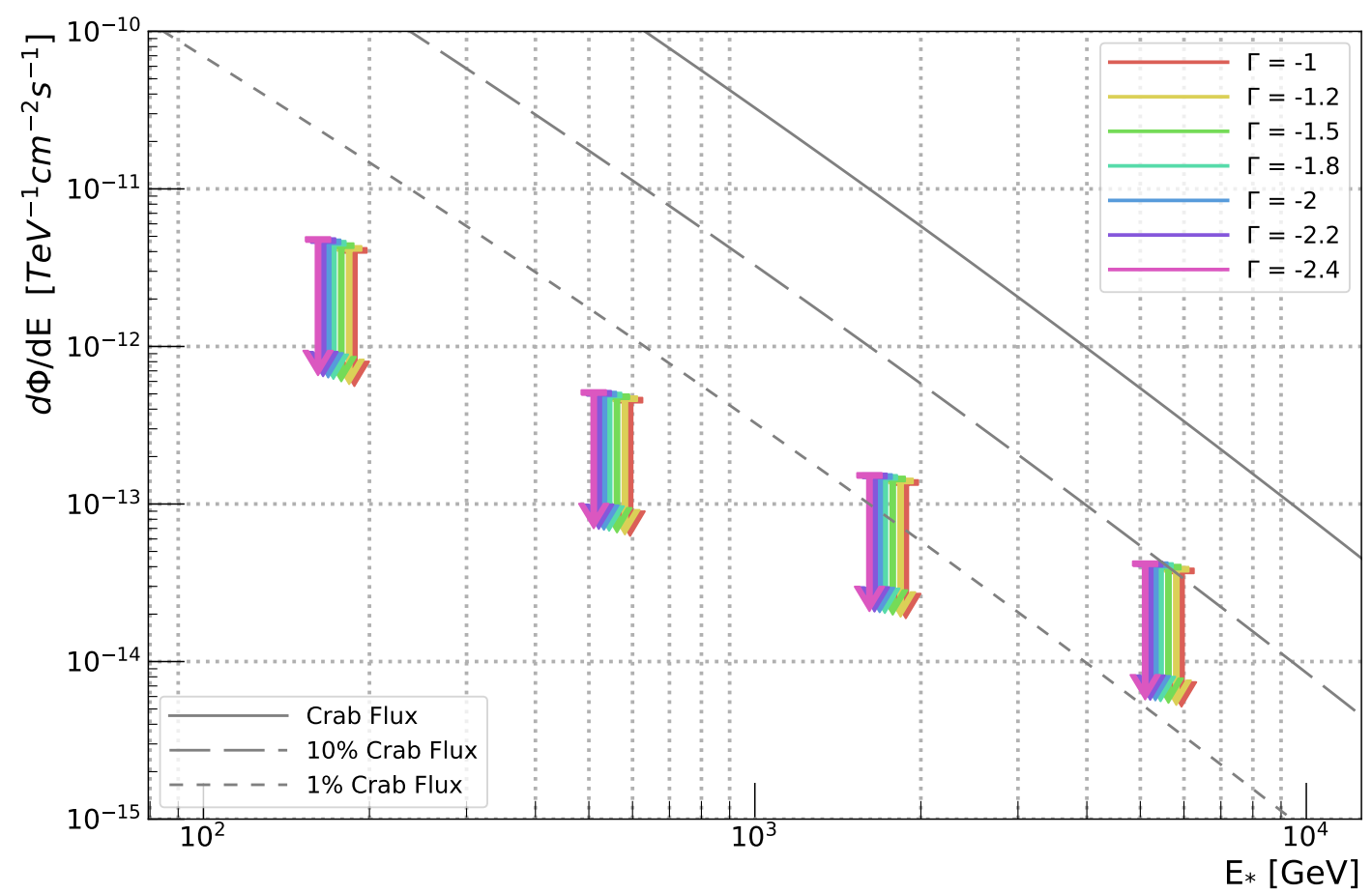

Figure 3: Differential gamma-ray flux upper limits of Tri II, assuming a point-like source and a power-law emission spectrum with various spectral slopes $\Gamma$. The solid gray line represents the Crab Nebula flux as detected by MAGIC, as well as the $10 \%$ and $1 \%$ of the Crab Nebula flux for reference. The x-axis is defined in terms of the pivot energy $E^{*}$, calculated for each energy bin (see Equation A.2 of [12]). The numerical values of the obtained flux ULs are shown in Appendix A

\subsection{Determination of $J_{\text {ann }}$}

Due to the lack of sufficient spectroscopic data for Tri II, a precise determination of $\rho(r)$, and hence $J_{\text {ann }}$ (through Eq. 3), cannot be made. Therefore, we estimate $J_{\text {ann }}$ with an alternative approach based on scaling relations among various observables inferred from other dSphs [27, 28].

Several studies suggest the existence of a number of scaling relations between various observable characteristics of dSphs and their DM content. It is observed that many dSphs tend to have similar integrated DM masses independent of their luminosities [29, 30, 31. This leads to a simple relation that links the heliocentric distance of the dSph $d$ to its value of $J_{\text {ann }}$, scaling as $J_{\text {ann }} \propto d^{-2}$ [32, 27]. Note that estimates on $d$ are generally derived with variable stars or isochrone fitting and do not rely on stellar kinematic information. Using statistical models which involve the fitting of parameters of a large number of dSphs, Pace \& Strigari [28] find that the best fit of the $J_{\text {ann }} \propto d^{-2}$ scaling relation is:

$$
\frac{J_{\mathrm{ann}}\left(0.5^{\circ}\right)}{\mathrm{GeV}^{2} \mathrm{~cm}^{-5}}=10^{18.30 \pm 0.07}\left(\frac{d}{100 \mathrm{kpc}}\right)^{-2}
$$

with an intrinsic scatter of $\sigma_{\log _{10} J_{\text {ann }}}=0.30 \pm 0.07$ and where $J_{\text {ann }}$ is integrated out to $0.5^{\circ}$ from the center of each dSph. Here, the intrinsic scatter quantifies the spread of the parent population around an average relation due to poorly constrained or unknown dependencies on physical parameters that characterize the phase space of the sample. We also note that the reasons for selecting a $J$-factor scaling relation which includes DM halos out to $0.5^{\circ}$ from the center of each dSph is twofold: first, Pace \& Strigari [28] find that 


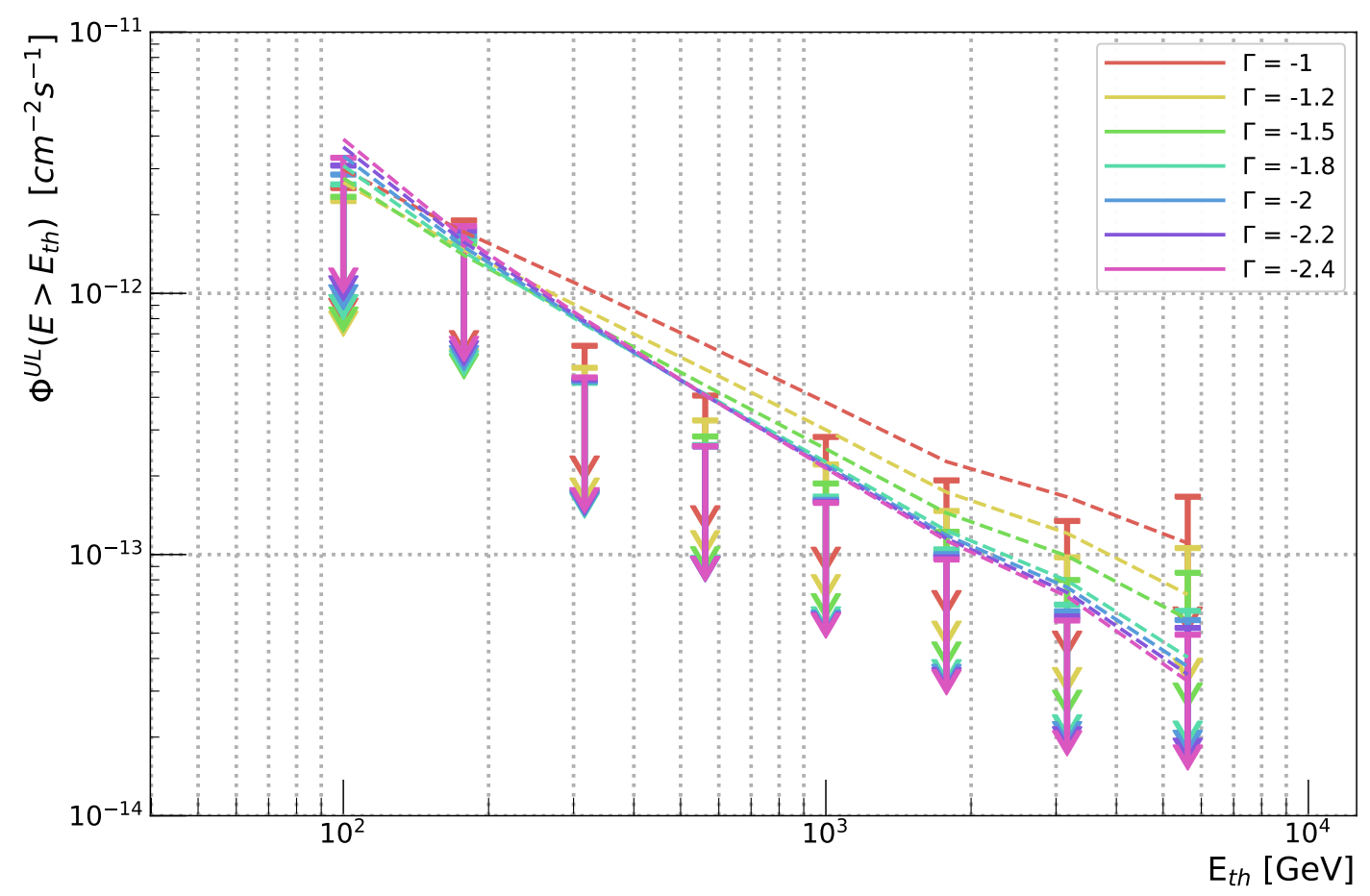

Figure 4: Integral gamma-ray flux upper limits of Tri II for events with energy $E>E_{\text {th }}$, assuming a point-like source and a power-law emission spectrum with various spectral slopes $\Gamma$. The 'null hypothesis' case is represented by dashed lines and the numerical values of the obtained flux ULs are shown in Appendix A

at this angle the scaling relation has the lowest scatter compared to a variety of other integration angles. Second, the majority of the DM content of the dSphs used to calculate the relation is contained within an integral angle of $0.5^{\circ}$ (e.g. see Table A2 of [28] and Table 2 of 33]).

Based on its distance of $d=30 \pm 2 \mathrm{kpc}$, Tri II is calculated to have a $J$-factor of $\log _{10}\left[J_{\text {ann }}\left(0.5^{\circ}\right) / \mathrm{GeV}^{2} \mathrm{~cm}^{-5}\right]=19.35 \pm 0.37$. The uncertainty on $J_{\text {ann }}$ is computed by adding the $1 \sigma$ extent of the intrinsic scatter to the error of Eq. 4 and must be treated carefully. We consider this uncertainty to be reasonable under the assumption that the physical properties of Tri II align with the dSph scaling relations in the literature. Note that this value for $J_{\text {ann }}$ is also consistent with other scaling relations, such as the luminosity scaling relation of [28].

In general, the DM distribution of a source can be reconstructed from good-quality spectroscopic information. Since this is not possible for Tri II based on predictions by numerical N-body DM simulations (as discussed in Section 2, we choose to estimate the DM density profile of Tri II.

We first adopt an Einasto profile [34] for the DM density of Tri II:

$$
\rho(r)=\rho_{-2} \exp \left\{-\frac{2}{\alpha}\left[\left(\frac{r}{r_{-2}}\right)^{\alpha}-1\right]\right\} .
$$

Here, the parameters $\rho_{-2}$ and $r_{-2}$ represent the density and radius at which the local slope of $\rho_{r}$ is equal to -2 , respectively. We fix the profile index $\alpha=0.16$. Such a choice is motivated by the results of the Aquarius Project, an ultrahigh-resolution particle simulation of MW-sized galactic halos [35].

In order to estimate the two unknown Einasto profile parameters $\rho_{-2}$ and $r_{-2}$, we employ two constraints: first, the integration of the profile along the l.o.s., as defined in Eq. 3. should reproduce the value of $J_{\text {ann }}$ 
provided by the scaling relations. The second constraint is related to the tidal radius $r_{t}$ of Tri II, or the radius at which the DM density of Tri II is equal to the local DM density of the MW halo $\rho_{\mathrm{DM}}^{\mathrm{MW}}$. This criterion can be used as a benchmark to describe the extent of MW subhalos [see e.g. 33]. In particular, we adopt equation 19 from [33]:

$$
\rho_{\mathrm{DM}}^{\operatorname{TriII}}\left(r_{t}\right)=\rho_{\mathrm{DM}}^{\mathrm{MW}}\left(d_{\mathrm{GC}}-r_{t}\right)
$$

where $d_{\mathrm{GC}}=36 \mathrm{kpc}$ is the galactocentric distance of Tri II 10 and $\rho_{\mathrm{DM}}^{\text {TriII }}\left(r_{t}\right)$ is the Einasto density of Tri II evaluated at its tidal radius. We adopt a value of $r_{t}=5_{-3}^{+1} \mathrm{kpc}$ for Tri II following [28], as this range represents the median $r_{t}$ of systems with unresolved kinematics in their sample. The density of the MW DM halo at the tidal radius of Tri II as determined by the Navarro-Frenk-White model of 36] is $\log _{10}\left[\rho_{\mathrm{DM}}^{\mathrm{MW}}\left(d_{\mathrm{GC}}-r_{t}\right) / \mathrm{M}_{\odot} \mathrm{kpc}^{-3}\right]=5.93_{-0.31}^{+0.23}$. We use Eqs. 3 and 6 to calculate $\rho_{-2}$ and $r_{-2}$, while fixing $J_{\text {ann }}, r_{t}$, and $\rho_{\mathrm{DM}}^{\mathrm{MW}}$. The integrals of Eq. 3 are computed using the CLUMPY code [37, 38, 39]. Solving this system numerically yields final values for the Einasto profile parameters of $\log _{10}\left[\rho_{-2} / \mathrm{M}_{\odot} \mathrm{kpc}^{-3}\right]=8.53_{-0.09}^{+1.50}$ and $r_{-2}=0.0714_{-0.0662}^{+0.0386} \mathrm{kpc}$. For this result, more than $86 \%$ of the total integrated $J$-factor signal is contained within the region $\theta^{2}=0.02 \mathrm{deg}^{2}$ about the center of the source.

\subsection{Likelihood analysis}

We used the full likelihood method, developed by [40] in conjunction with the binned likelihood analysis scheme presented in [13]. The DM signal was modeled with the PYTHIA simulation package version 8.135 with electroweak corrections 41. The likelihood $\mathcal{L}$ is the product of two likelihood functions, one for each telescope pointing direction $i=1,2$. The binned likelihood for each pointing is written as:

$$
\begin{aligned}
\mathcal{L}_{i}= & \mathcal{L}_{i}\left(\langle\sigma v\rangle ;\left\{b_{i j}\right\}_{j=1, \ldots, N_{\mathrm{bins}}}, J_{\mathrm{ann}}, \tau_{i} \mid\left(N_{\mathrm{ON}, i j}, N_{\mathrm{OFF}, i j}\right)_{j=1, \ldots, N_{\mathrm{bins}}}\right) \\
= & \prod_{j=1}^{N_{\mathrm{bins}}}\left[\frac{\left(g_{i j}(\langle\sigma v\rangle)+b_{i j}\right)^{N_{\mathrm{ON}, i j}}}{N_{\mathrm{ON}, i j} !} e^{-\left(g_{i j}(\langle\sigma v\rangle)+b_{i j}\right)}\right. \\
& \left.\times \frac{\left(\tau_{i} b_{i j}\right)^{N_{\mathrm{OFF}, i j}}}{N_{\mathrm{OFF}, i j} !} e^{-\left(\tau_{i} b_{i j}\right)}\right] \\
& \times \mathcal{T}\left(\tau_{i} \mid \tau_{\mathrm{obs}, i}, \sigma_{\tau, i}\right) \times \mathcal{J}\left(J_{\mathrm{ann}} \mid J_{\mathrm{obs}}, \sigma_{\log _{10} J_{\mathrm{obs}}}\right)
\end{aligned}
$$

where

- $N_{\text {bins }}$ is the number of considered bins of the estimated energy;

- $g_{i j}$ and $b_{i j}$ are the estimated number of signal and background events respectively;

- $N_{\mathrm{ON}, i j}$ and $N_{\mathrm{OFF}, i j}$ are the number of observed events in the $j$-th ON and OFF bins respectively, where OFF events are obtained from the analogous ON region at the complementary pointing;

- $\mathcal{J}$ is the likelihood for the annihilation J-factor $J_{a n n}$, given measured $\log _{10} J_{o b s}$ and its uncertainty $\sigma_{\log _{10} J_{\text {obs }}}$, as defined by the equation

$$
\mathcal{J}\left(J_{\mathrm{ann}} \mid J_{\mathrm{obs}}, \sigma_{\log _{10} J_{\text {obs }}}\right)=\frac{1}{\ln (10) J_{\mathrm{obs}} \sqrt{2 \pi} \sigma_{\log _{10} J_{\mathrm{obs}}}} \times e^{-\left(\log _{10}\left(J_{\mathrm{ann}}\right)-\log _{10}\left(J_{\mathrm{obs}}\right)\right)^{2} / 2 \sigma_{\log _{10} J_{\mathrm{obs}}}^{2}} ;
$$

- $\mathcal{T}$ is the likelihood for $\tau_{i}$ (the $\mathrm{ON} / \mathrm{OFF}$ ) acceptance ratio, parameterized by a Gaussian function with mean $\tau_{\mathrm{obs}, i}$ and variance $\sigma_{\tau, i}^{2}$, which includes statistical and systematic uncertainties, added in quadrature assuming Poisson statistics. We consider a systematic uncertainty for the parameter $\tau_{\mathrm{sys}, i}=0.015 \tau_{\mathrm{obs}, i}$, a value that has been established in [12]. 
The parameters $J_{\mathrm{ann}}, \tau_{i}$, and $b_{i j}$ are nuisance parameters. The variable $g_{i j}$ depends on the free parameter $\langle\sigma v\rangle$ as such:

$$
g_{i j}(\langle\sigma v\rangle)=T_{\mathrm{obs}, i} \int_{E_{\min , j}^{\prime}}^{E_{\max , j}^{\prime}} d E^{\prime} \int_{0}^{\infty} d E \frac{d \Phi_{\gamma}(\langle\sigma v\rangle)}{d E} A_{\mathrm{eff}}(E) G\left(E^{\prime} \mid E\right),
$$

where $T_{\mathrm{obs}, i}$ is the total observation time, and $E$ and $E^{\prime}$ are the true and estimated gamma-ray energy, respectively. $E_{\min , j}^{\prime}$ and $E_{\max , j}^{\prime}$ are the minimum and maximum energies of the j-th energy bin, respectively. $A_{\text {eff }}$ is the effective collection area, and $G$ represents the probability density function of the energy estimator, both computed from a MC simulated gamma-ray data set following the spatial distribution expected from signals induced by the annihilation of DM in Tri II computed in Section 3.1 (through a MAGIC dedicated procedure described in [13]).

\subsection{Results on dark matter annihilation models}

We performed a search for annihilating DM in the candidate dSph Tri II using 62.4 h of good-quality data, assuming DM particles with masses between $200 \mathrm{GeV}$ and $200 \mathrm{TeV}$, and a $100 \%$ branching ratio into four different channels: $\mathrm{b} \overline{\mathrm{b}}, \mu^{+} \mu^{-}, \tau^{+} \tau^{-}$, and $\mathrm{W}^{+} \mathrm{W}^{-}$. We present the $95 \% \mathrm{CL}$ ULs on the thermallyaveraged DM annihilation cross section $\left\langle\sigma_{\text {ann }} v\right\rangle$ for each annihilation channel with a binned likelihood analysis ${ }^{2}$ with 10 logarithmically-spaced bins ranging from $80 \mathrm{GeV}$ to $10 \mathrm{TeV} \mathrm{S}^{3}$ We considered a $J$-factor of $\log _{10}\left[J_{\text {ann }}\left(0.5^{\circ}\right) / \mathrm{GeV}^{2} \mathrm{~cm}^{-5}\right]=19.35 \pm 0.37$ as obtained in Section 3.1. Results for leptonic and hadronic channels are shown in Figure 5 .

The expected limits from the null hypothesis of $\langle\sigma v\rangle=0$ (dashed lines) and its $68 \%$ and $95 \%$ containment bands (in green and yellow, respectively) are also shown. These bands are calculated with 300 MC simulations in which both $\mathrm{ON}$ and $\mathrm{OFF}$ regions are generated from pure background probability density functions while assuming a similar exposure for the real data. In this case, $\tau_{i}$ is taken as a nuisance parameter in the likelihood function. Our limits are consistent with the null hypothesis for all considered DM models. The most constraining limits are $\langle\sigma v\rangle^{\mathrm{UL}}=1.53 \times 10^{-23} \mathrm{~cm}^{3} \mathrm{~s}^{-1}$ in the $\mathrm{b} \overline{\mathrm{b}}$ channel for $m_{D M}=500 \mathrm{GeV}$ and $\langle\sigma v\rangle^{\mathrm{UL}}=3.05 \times 10^{-24} \mathrm{~cm}^{3} \mathrm{~s}^{-1}$ in the $\tau^{+} \tau^{-}$channel for $m_{D M}=120 \mathrm{GeV}$. The limits assuming a fixed $J_{\text {ann }}$ with no uncertainly, calculated by ignoring the $J$-factor term in Eq. 7) are also shown (dotted lines).

The Tri II results for the $\tau^{+} \tau^{-}$channel are compatible with previous MAGIC dSph studies, including those derived from $94.8 \mathrm{~h}$ and $157.9 \mathrm{~h}$ of Ursa Major II [13] and Segue I [12] observations, respectively. Due to the difference in the analysis method developed and adopted after the Segue I results, in both the treatment of the $J$-factor as a nuisance parameter and in the background modeling methods, a straightforward comparison is not easily achievable. However, a comparison is made possible by looking at the MAGIC-only Segue 1 results shown in a later combined analysis between Fermi and MAGIC [14 using the same data set. The differences with respect to this later work can be explained taking into account the difference in exposures and $J$-factor between both analyses. Results from different dSphs can be combined into a unique limit using a specific recipe described in [40]. Some preliminary results were shown in [42] and will be published in a future manuscript.

\section{Summary and Conclusions}

An observation campaign of Triangulum II was carried out with MAGIC starting in August 2016 as a reaction to the announcement of a potential large quantity of DM content within the source [10. A total of $62.4 \mathrm{~h}$ of high-quality data were collected, and no significant gamma-ray signal was detected in both the standard point-like and extended-profile DM analyses. Integral and differential upper limits on the gammaray flux were obtained for the first time for the region of Tri II. These limits can be used to guide future searches in the source region, and act as a baseline for further studies with next generation IACTs [43].

\footnotetext{
${ }^{2}$ We construct and minimize our likelihood making use of https://github.com/javierrico/gLike

${ }^{3}$ Empty bins were merged with neighboring ones.
} 

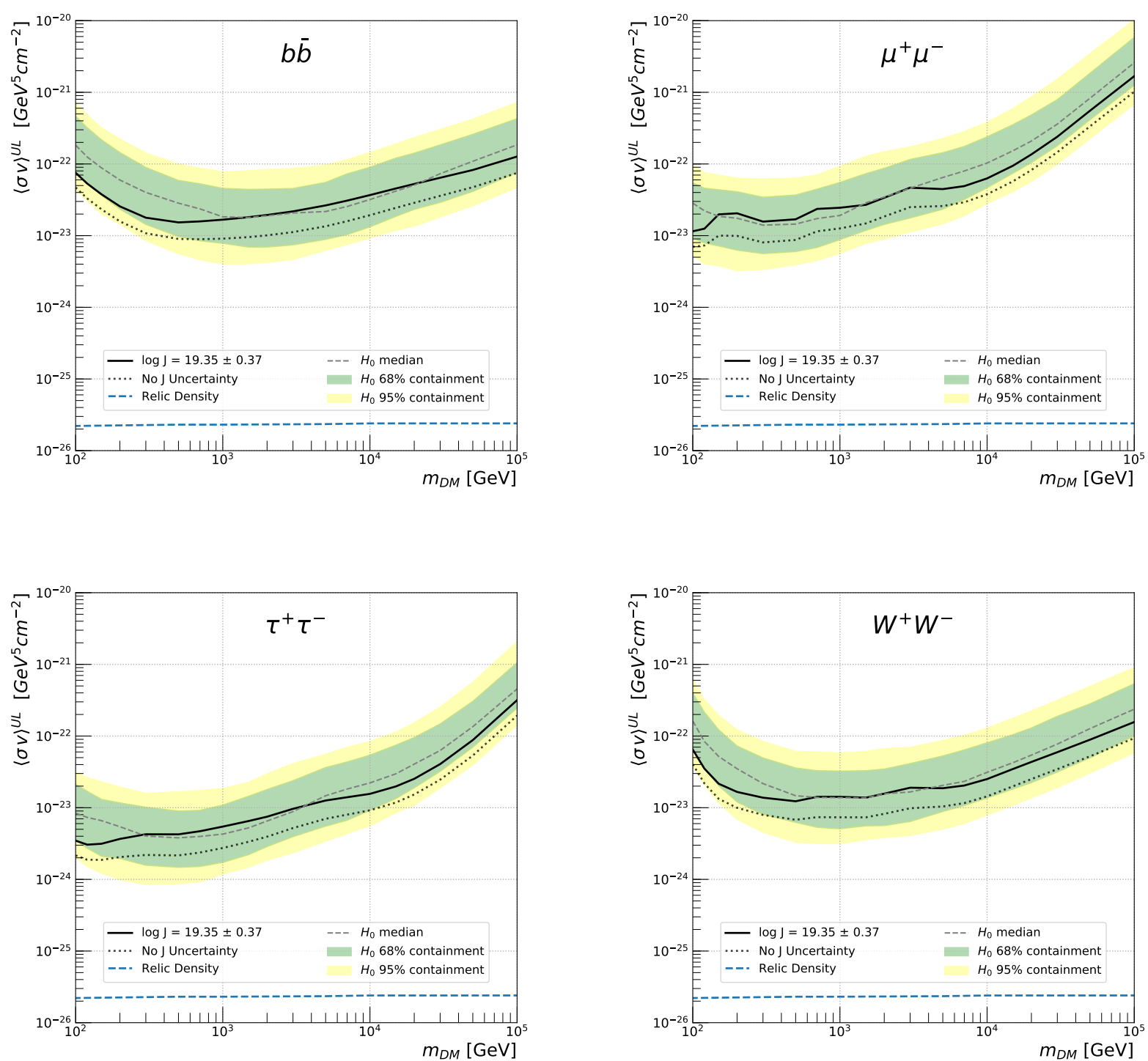

Figure 5: 95\% CL ULs on the DM annihilation cross section $\langle\sigma v\rangle$ as a function of the dark matter mass $m_{D M}$ for four different channels: $\mathrm{b} \overline{\mathrm{b}}$ (upper left), $\mu^{+} \mu^{-}$(upper right), $\tau^{+} \tau^{-}$(bottom left), and $\mathrm{W}^{+} \mathrm{W}^{-}$(bottom right). The extended $J$-factor profile calculated in Section 3.1, with a total value of $\log \left[J_{\text {ann }}\left(0.5^{\circ}\right) / \mathrm{GeV}^{2} \mathrm{~cm}^{-5}\right]=19.35 \pm 0.37$, is used in the likelihood to calculate the limits. The observed (solid line) and the expected (dashed line) limits are shown together with the $1 \sigma$ (green) and $2 \sigma$ (yellow) bands. The dotted line shows the limits calculated with the same $J$-factor but assuming no uncertainty. The thermal relic cross section (blue dash-dotted) is also shown [4.

A dedicated search for an extended DM source was carried out in the region. Due to the limited available stellar kinematic data for Tri II, a method based on scaling relations was used to infer the DM content of the object, resulting in a $J$-factor of $\log _{10}\left[J_{\text {ann }}\left(0.5^{\circ}\right) / \mathrm{GeV}^{2} \mathrm{~cm}^{-5}\right]=19.35 \pm 0.37$. Based on knowledge provided by numerical simulations, we derived a DM profile for Tri II which we subsequently used in a rigorous DM binned likelihood analysis. The obtained $95 \%$ confidence level upper limits on the DM annihilation cross section are compatible with the null hypothesis $68 \%$ containment bands. In addition, the use of the 
maximum likelihood method would allow for the combination of our data with those available for additional dSphs, even if observed with other instruments. These combined analyses will help to improve the current sensitivity of DM searches towards the thermal relic limit in the $>1 \mathrm{TeV}$ mass range [e.g. 14].

With the sparse amount of empirical knowledge surrounding the DM content of Tri II presently available in the literature, we believe an approach that does not rely on spectroscopic data is currently the most reliable option. Ideally, in the near future, a more precise determination of the DM annihilation limits derived from observations of Tri II can be obtained using the same MAGIC data set as soon as the behavior of its stellar kinematics is better understood. 


\section{Appendix A. Gamma-ray flux upper limits}

Tables A.1 and A.2 show the differential and integral upper limits, respectively, on the gamma-ray flux from Tri II assuming a point-like source and power-law spectra with various photon indices $\Gamma$. For more details see Section 2.1 . In both tables, $N_{\mathrm{ON}}$ and $N_{\mathrm{OFF}}$ are the total number of observed events in the ON and OFF regions, respectively. The normalization factor between the ON and OFF regions is represented by $\tau$. Finally, $N_{\mathrm{ex}}^{\mathrm{UL}}$ is the upper limit on the number of excess events calculated for a 95\% CL with the conventional method [4], assuming a systematic uncertainty on the overall detection efficiency of 30\% (a standard value for MAGIC analyses).

\begin{tabular}{cccccccccccc}
\hline & & & & & \multicolumn{5}{c}{$d \Phi / d E^{\mathrm{UL}}\left[\mathrm{TeV}^{-1} \mathrm{~cm}^{-2} \mathrm{~s}^{-1}\right]$} \\
\cline { 6 - 11 }$\Delta E[\mathrm{GeV}]$ & $N_{\mathrm{ON}}$ & $N_{\mathrm{OFF}}$ & $\tau$ & $N_{\mathrm{ex}}^{\mathrm{UL}}$ & $\Gamma=-1.0$ & $\Gamma=-1.2$ & $\Gamma=-1.5$ & $\Gamma=-1.8$ & $\Gamma=-2$ & $\Gamma=-2.2$ & $\Gamma=-2.4$ \\
\hline $100-316.2$ & 6854 & 6844 & 1.002 & 309.3 & $4.1 \times 10^{-12}$ & $4.2 \times 10^{-12}$ & $4.4 \times 10^{-12}$ & $4.5 \times 10^{-12}$ & $4.6 \times 10^{-12}$ & $4.7 \times 10^{-12}$ & $4.8 \times 10^{-12}$ \\
$316.2-1000$ & 967 & 1003 & 1.011 & 71.8 & $4.6 \times 10^{-13}$ & $4.7 \times 10^{-13}$ & $4.8 \times 10^{-13}$ & $4.9 \times 10^{-13}$ & $5.0 \times 10^{-13}$ & $5.1 \times 10^{-13}$ & $5.1 \times 10^{-13}$ \\
$1000-3162.2$ & 113 & 120 & 1.005 & 28.4 & $1.4 \times 10^{-13}$ & $1.4 \times 10^{-13}$ & $1.4 \times 10^{-13}$ & $1.5 \times 10^{-13}$ & $1.5 \times 10^{-13}$ & $1.5 \times 10^{-13}$ & $1.5 \times 10^{-13}$ \\
$3162.2-10000$ & 7 & 9 & 1.005 & 7.4 & $3.8 \times 10^{-14}$ & $3.9 \times 10^{-14}$ & $4.0 \times 10^{-14}$ & $4.1 \times 10^{-14}$ & $4.1 \times 10^{-14}$ & $4.2 \times 10^{-14}$ & $4.2 \times 10^{-14}$ \\
\hline
\end{tabular}

Table A.1: Differential gamma-ray flux upper limits of Tri II

\begin{tabular}{|c|c|c|c|c|c|c|c|c|c|c|c|}
\hline \multirow[b]{2}{*}{$E_{\mathrm{th}}[\mathrm{GeV}]$} & \multirow[b]{2}{*}{$N_{\mathrm{ON}}$} & \multirow[b]{2}{*}{$N_{\mathrm{OFF}}$} & \multirow[b]{2}{*}{$\tau$} & \multirow[b]{2}{*}{$N_{\mathrm{ex}}^{\mathrm{UL}}$} & \multicolumn{7}{|c|}{$\Phi^{\mathrm{UL}}\left[\mathrm{cm}^{-2} \mathrm{~s}^{-1}\right]$} \\
\hline & & & & & $\Gamma=-1.0$ & $\Gamma=-1.2$ & $\Gamma=-1.5$ & $\Gamma=-1.8$ & $\Gamma=-2$ & $\Gamma=-2.2$ & $\Gamma=-2.4$ \\
\hline 100 & 7942 & 7978 & 1.002 & 269.3 & $2.5 \times 10^{-12}$ & $2.3 \times 10^{-12}$ & $2.3 \times 10^{-12}$ & $2.6 \times 10^{-12}$ & $2.9 \times 10^{-12}$ & $3.1 \times 10^{-12}$ & $3.3 \times 10^{-12}$ \\
\hline 177.8 & 2930 & 2918 & 1.002 & 211.3 & $1.9 \times 10^{-12}$ & $1.6 \times 10^{-12}$ & $1.6 \times 10^{-12}$ & $1.6 \times 10^{-12}$ & $1.7 \times 10^{-12}$ & $1.7 \times 10^{-12}$ & $1.8 \times 10^{-12}$ \\
\hline 316.2 & 1088 & 1133 & 1.011 & 70.9 & $6.3 \times 10^{-13}$ & $5.2 \times 10^{-13}$ & $4.7 \times 10^{-13}$ & $4.5 \times 10^{-13}$ & $4.6 \times 10^{-13}$ & $4.7 \times 10^{-13}$ & $4.8 \times 10^{-13}$ \\
\hline 562.3 & 380 & 402 & 1.008 & 44.5 & $4.1 \times 10^{-13}$ & $3.3 \times 10^{-13}$ & $2.8 \times 10^{-13}$ & $2.6 \times 10^{-13}$ & $2.6 \times 10^{-13}$ & $2.6 \times 10^{-13}$ & $2.6 \times 10^{-13}$ \\
\hline 1000 & 121 & 130 & 1.005 & 28.6 & $2.8 \times 10^{-13}$ & $2.2 \times 10^{-13}$ & $1.9 \times 10^{-13}$ & $1.7 \times 10^{-13}$ & $1.6 \times 10^{-13}$ & $1.6 \times 10^{-13}$ & $1.6 \times 10^{-13}$ \\
\hline 1778.3 & 29 & 31 & 1.005 & 16.6 & $1.9 \times 10^{-13}$ & $1.5 \times 10^{-13}$ & $1.2 \times 10^{-13}$ & $1.1 \times 10^{-13}$ & $1.0 \times 10^{-13}$ & $9.8 \times 10^{-14}$ & $9.6 \times 10^{-14}$ \\
\hline 3162.3 & 8 & 9 & 1.005 & 8.9 & $1.3 \times 10^{-13}$ & $9.8 \times 10^{-14}$ & $8.0 \times 10^{-14}$ & $6.4 \times 10^{-14}$ & $6.1 \times 10^{-14}$ & $5.8 \times 10^{-14}$ & $5.6 \times 10^{-14}$ \\
\hline 5623.4 & 2 & 1 & 1.000 & 6.7 & $1.7 \times 10^{-13}$ & $1.1 \times 10^{-13}$ & $8.5 \times 10^{-14}$ & $6.1 \times 10^{-14}$ & $5.6 \times 10^{-14}$ & $5.2 \times 10^{-14}$ & $4.9 \times 10^{-14}$ \\
\hline
\end{tabular}

Table A.2: Integral gamma-ray flux upper limits of Tri II for events with energies above various energy thresholds $E_{\mathrm{th}}$. 


\section{Acknowledgments}

We would like to thank Miguel Angel Sánchez-Conde for his assistance interpreting the Aquarius Project simulations.

We would also like to thank the Instituto de Astrofísica de Canarias for the excellent working conditions at the Observatorio del Roque de los Muchachos in La Palma. The financial support of the German BMBF and MPG, the Italian INFN and INAF, the Swiss National Fund SNF, the ERDF under the Spanish MINECO (FPA2017-87859-P, FPA2017-85668-P, FPA2017-82729-C6-2-R, FPA201782729-C6-6-R, FPA2017-82729-C6-5-R, AYA2015-71042-P, AYA2016-76012-C3-1-P, ESP2017-87055-C2-2-P, FPA2017âĂ $\check{R} 90566 \hat{A} \breve{A}$ REEDC), the Indian Department of Atomic Energy, the Japanese JSPS and MEXT, the Bulgarian Ministry of Education and Science, National RI Roadmap Project DO1-153/28.08.2018 and the Academy of Finland grant nr. 320045 is gratefully acknowledged. This work was also supported by the Spanish Centro de Excelencia "Severo Ochoa" SEV-2016-0588 and SEV-2015-0548, and Unidad de Excelencia "María de Maeztu" MDM-2014-0369, by the Croatian Science Foundation (HrZZ) Project IP-2016-06-9782 and the University of Rijeka Project 13.12.1.3.02, by the DFG Collaborative Research Centers SFB823/C4 and SFB876/C3, the Polish National Research Centre grant UMO-2016/22/M/ST9/00382 and by the Brazilian MCTIC, CNPq and FAPERJ.

\section{References}

[1] M. Roos, Dark Matter: The evidence from astronomy, astrophysics and cosmology, arXiv e-prints (2010) arXiv:1001.0316 (Jan 2010). arXiv:1001.0316.

[2] K. Freese, Review of Observational Evidence for Dark Matter in the Universe and in upcoming searches for Dark Stars, in: E. Pécontal, T. Buchert, P. di Stefano, Y. Copin (Eds.), EAS Publications Series, Vol. 36 of EAS Publications Series, 2009, pp. 113-126 (Jan 2009). arXiv:0812.4005 doi:10.1051/eas/0936016

[3] P. Hut, Limits on masses and number of neutral weakly interacting particles, Physics Letters B 69 (1977) 85-88 (Jul 1977). doi:10.1016/0370-2693(77)90139-3.

[4] G. Steigman, B. Dasgupta, J. F. Beacom, Precise relic WIMP abundance and its impact on searches for dark matter annihilation, Phys. Rev. D86 (2012) 023506 (Jul 2012). arXiv:1204.3622, doi:10.1103/PhysRevD.86.023506

[5] Planck Collaboration, et al., Planck 2018 results. VI. Cosmological parameters, arXiv e-prints (2018) arXiv:1807.06209 (Jul 2018). arXiv:1807.06209

[6] J. Aleksić, et al., The major upgrade of the MAGIC telescopes, Part I: The hardware improvements and the commissioning of the system, Astroparticle Physics 72 (2016) 61-75 (Jan 2016). arXiv:1409.6073, doi:10.1016/j.astropartphys.2015. 04.004

[7] M. Doro, A decade of dark matter searches with ground-based Cherenkov telescopes, Nuclear Instruments and Methods in Physics Research A 742 (2014) 99-106 (Apr 2014). arXiv:1404.5017, doi:10.1016/j.nima.2013.12.010

[8] P. Ullio, M. Valli, A critical reassessment of particle Dark Matter limits from dwarf satellites, Journal of Cosmology and Astro-Particle Physics 2016 (2016) 025 (Jul 2016). arXiv:1603.07721 doi:10.1088/1475-7516/2016/07/025

[9] L. E. Strigari, et al., The Most Dark-Matter-dominated Galaxies: Predicted Gamma-Ray Signals from the Faintest Milky Way Dwarfs, ApJ678 (2008) 614-620 (May 2008). arXiv:0709.1510 doi:10.1086/529488

[10] E. N. Kirby, J. G. Cohen, J. D. Simon, P. Guhathakurta, Triangulum II: Possibly a Very Dense Ultra-faint Dwarf Galaxy, ApJ814 (2015) L7 (Nov 2015). doi:10.1088/2041-8205/814/1/L7

[11] E. N. Kirby, J. G. Cohen, J. D. Simon, P. Guhathakurta, A. O. Thygesen, G. E. Duggan, Triangulum II. Not Especially Dense After All, ApJ838 (2017) 83 (Apr 2017). doi:10.3847/1538-4357/aa6570

[12] J. Aleksić, et al., Optimized dark matter searches in deep observations of Segue 1 with MAGIC, Journal of Cosmology and Astro-Particle Physics 2014 (2014) 008 (Feb 2014). arXiv:1312.1535 doi:10.1088/1475-7516/2014/02/008

[13] M. L. Ahnen, et al., Indirect dark matter searches in the dwarf satellite galaxy Ursa Major II with the MAGIC telescopes, Journal of Cosmology and Astro-Particle Physics 2018 (2018) 009 (Mar 2018). arXiv:1712.03095, doi: $10.1088 / 1475-7516 / 2018 / 03 / 009$.

[14] M. L. Ahnen, et al., Limits to dark matter annihilation cross-section from a combined analysis of MAGIC and Fermi-LAT observations of dwarf satellite galaxies, Journal of Cosmology and Astro-Particle Physics 2016 (2016) 039 (Feb 2016). arXiv: 1601.06590 , doi: 10.1088/1475-7516/2016/02/039

[15] N. Kaiser, et al., The Pan-STARRS wide-field optical/NIR imaging survey, in: Ground-based and Airborne Telescopes III, Vol. 7733, 2010, p. 77330E (Jul 2010). doi:10.1117/12.859188

[16] B. P. M. Laevens, et al., A New Faint Milky Way Satellite Discovered in the Pan-STARRS1 $3 \pi$ Survey, ApJ802 (2015) L18 (Apr 2015). doi:10.1088/2041-8205/802/2/L18

[17] V. P. Fomin, A. A. Stepanian, R. C. Lamb, D. A. Lewis, M. Punch, T. C. Weekes, New methods of atmospheric Cherenkov imaging for gamma-ray astronomy. I. The false source method, Astroparticle Physics 2 (1994) 137-150 (May 1994). doi:10.1016/0927-6505(94)90036-1

[18] N. F. Martin, et al., Triangulum II: A Very Metal-poor and Dynamically Hot Stellar System, ApJ818 (2016) 40 (Feb 2016). doi:10.3847/0004-637X/818/1/40 
[19] S. o. Faber, The DEIMOS spectrograph for the Keck II Telescope: integration and testing, in: Instrument Design and Performance for Optical/Infrared Ground-based Telescopes, Vol. 4841, 2003, pp. 1657-1669 (Mar 2003). doi:10.1117/ 12.460346

[20] R. Zanin, et al., MARS, the MAGIC analysis and reconstruction software, in: Proceedings of the 33rd International Cosmic Ray Conference (ICRC2013): Rio de Janeiro, Brazil, July 2-9, 2013, 2013, p. 0773 (2013). URL http://inspirehep.net/record/1412925/files/icrc2013-0773.pdf

[21] C. Fruck, et al., A novel LIDAR-based Atmospheric Calibration Method for Improving the Data Analysis of MAGIC, arXiv e-prints (2014) arXiv:1403.3591 (Mar 2014). arXiv:1403.3591.

[22] J. Albert, et al., Implementation of the Random Forest method for the Imaging Atmospheric Cherenkov Telescope MAGIC, Nuclear Instruments and Methods in Physics Research A 588 (2008) 424-432 (Apr 2008). arXiv:0709.3719, doi:10.1016/ j.nima.2007.11.068

[23] T.-P. Li, Y.-Q. Ma, Analysis methods for results in gamma-ray astronomy., ApJ272 (1983) 317-324 (Sep 1983). doi: 10.1086/161295

[24] S. Lombardi, Advanced stereoscopic gamma-ray shower analysis with the MAGIC telescopes, International Cosmic Ray Conference 3 (2011) 266 (Jan 2011). arXiv:1109.6195, doi:10.7529/ICRC2011/V03/1150

[25] J. Albert, et al., VHE $\gamma$-Ray Observation of the Crab Nebula and its Pulsar with the MAGIC Telescope, ApJ674 (2008) 1037-1055 (Feb 2008). arXiv:0705.3244 doi:10.1086/525270

[26] L. Bergström, P. Ullio, J. H. Buckley, Observability of $\gamma$ rays from dark matter neutralino annihilations in the Milky Way halo, Astroparticle Physics 9 (1998) 137-162 (Aug 1998). arXiv:astro-ph/9712318, doi:10.1016/S0927-6505(98) 00015-2

[27] A. Albert, et al., Searching for Dark Matter Annihilation in Recently Discovered Milky Way Satellites with Fermi-Lat, ApJ834 (2017) 110 (Jan 2017). arXiv:1611.03184 doi:10.3847/1538-4357/834/2/110

[28] A. B. Pace, L. E. Strigari, Scaling relations for dark matter annihilation and decay profiles in dwarf spheroidal galaxies, MNRAS482 (2019) 3480-3496 (Jan 2019). arXiv:1802.06811 doi:10.1093/mnras/sty2839

[29] L. E. Strigari, et al., A common mass scale for satellite galaxies of the Milky Way, Nature454 (2008) 1096-1097 (Aug 2008). arXiv:0808.3772 doi:10.1038/nature07222

[30] M. G. Walker, M. Mateo, E. W. Olszewski, J. Peñarrubia, N. W. Evans, G. Gilmore, A Universal Mass Profile for Dwarf Spheroidal Galaxies?, ApJ704 (2009) 1274-1287 (Oct 2009). arXiv:0906.0341. doi:10.1088/0004-637X/704/2/1274

[31] J. Wolf, et al., Accurate masses for dispersion-supported galaxies, MNRAS406 (2010) 1220-1237 (Aug 2010). arXiv: 0908.2995 doi:10.1111/j.1365-2966.2010.16753.x.

[32] A. Drlica-Wagner, et al., Search for Gamma-Ray Emission from DES Dwarf Spheroidal Galaxy Candidates with Fermi-LAT Data, ApJ809 (2015) L4 (Aug 2015). arXiv:1503.02632, doi:10.1088/2041-8205/809/1/L4

[33] V. Bonnivard, et al., Dark matter annihilation and decay in dwarf spheroidal galaxies: the classical and ultrafaint dSphs, MNRAS453 (1) (2015) 849-867 (Oct 2015). arXiv:1504.02048 doi:10.1093/mnras/stv1601

[34] J. Einasto, On the Construction of a Composite Model for the Galaxy and on the Determination of the System of Galactic Parameters, Trudy Astrofizicheskogo Instituta Alma-Ata 5 (1965) 87-100 (Jan 1965).

[35] V. Springel, et al., The Aquarius Project: the subhaloes of galactic haloes, MNRAS391 (2008) 1685-1711 (Dec 2008). arXiv:0809.0898 doi:10.1111/j.1365-2966.2008.14066.x

[36] F. Nesti, P. Salucci, The Dark Matter halo of the Milky Way, AD 2013, Journal of Cosmology and Astro-Particle Physics 2013 (2013) 016 (Jul 2013). arXiv:1304.5127 doi:10.1088/1475-7516/2013/07/016

[37] A. Charbonnier, C. Combet, D. Maurin, CLUMPY: A code for $\gamma$-ray signals from dark matter structures, Computer Physics Communications 183 (3) (2012) 656-668 (Mar 2012). arXiv:1201.4728 doi:10.1016/j.cpc.2011.10.017

[38] V. Bonnivard, M. Hütten, E. Nezri, A. Charbonnier, C. Combet, D. Maurin, CLUMPY: Jeans analysis, $\gamma$-ray and $\nu$ fluxes from dark matter (sub-)structures, Computer Physics Communications 200 (2016) 336-349 (Mar 2016). arXiv:1506.07628, doi:10.1016/j.cpc.2015.11.012.

[39] M. Hütten, C. Combet, D. Maurin, CLUMPY v3: $\gamma$-ray and $\nu$ signals from dark matter at all scales, Computer Physics Communications 235 (2019) 336-345 (Feb 2019). arXiv:1806.08639 doi:10.1016/j.cpc.2018.10.001

[40] J. Aleksić, J. Rico, M. Martinez, Optimized analysis method for indirect dark matter searches with imaging air Cherenkov telescopes, Journal of Cosmology and Astro-Particle Physics 2012 (2012) 032 (Oct 2012). arXiv:1209.5589, doi:10.1088/ $1475-7516 / 2012 / 10 / 032$

[41] M. Cirelli, et al., PPPC 4 DM ID: a poor particle physicist cookbook for dark matter indirect detection, Journal of Cosmology and Astro-Particle Physics 2011 (2011) 051 (Mar 2011). arXiv:1012.4515, doi:10.1088/1475-7516/2011/03/ 051 .

[42] L. Oakes, Combined Dark Matter Searches Towards Dwarf Spheroidal Galaxies with Fermi-LAT, HAWC, HESS, MAGIC and VERITAS, in: 36th International Cosmic Ray Conference (ICRC2019), Vol. 36 of International Cosmic Ray Conference, 2019, p. 539 (Jul 2019). arXiv:1909.06310

[43] Cherenkov Telescope Array Consortium, et al., Science with the Cherenkov Telescope Array, 2019 (2019). doi:10.1142/ 10986

[44] W. A. Rolke, A. M. López, J. Conrad, Limits and confidence intervals in the presence of nuisance parameters, Nuclear Instruments and Methods in Physics Research A 551 (2005) 493-503 (Oct 2005). arXiv:physics/0403059 doi:10.1016/ j.nima.2005.05.068 